\title{
Rapid cessation of acute diarrhea using a novel solution of bioactive polyphenols: a randomized trial in Nicaraguan children
}

Arthur s. Dover, Neema Patel, KT Park

Goal We assessed the effectiveness of bioactive polyphenols contained in solution (LX) to restore normal bowel function in pediatric patients with acute diarrhea. Background While providing oral rehydration solution (ORS) is standard treatment for diarrhea in developing countries, plant-derived products have been shown to positively affect intestinal function. If a supplement to ORS resolves diarrhea more rapidly than ORS alone, it is an improvement to current care. Study In a randomized, double-blind, placebocontrolled cross-over study, 61 pediatric patients with uncontrolled diarrhea were randomized to receive either ORS+LX on day 1 and then ORS+water on day 2 (study arm) or ORS+water on day 1 and then ORS+LX on day 2 (control arm). Time to resolution and number of bowel movements were recorded. Results On day 1, the mean time to diarrhea resolution was 3.1 hours (study arm) versus 9.2 hours (control arm) $(p=0.002)$. In the study arm, $60 \%$ of patients had normal stool at their first bowel movement after consumption of the phenolic redoxigen solution (LX). On day 2, patients in the study arm continued to have normal stool while patients in the control arm achieved normal stool within 24 hours after consuming the test solution. Patients in the control arm experienced a reduction in the mean number of bowel movements from day 1 to day 2 after consuming the test solution $(p=0.0001)$. No adverse events were observed. Conclusions Significant decreases in bowel movement frequency and rapid normalization of stool consistency were observed with consumption of this novel solution. 


\section{Rapid Cessation of Acute Diarrhea Using a Novel Solution of Bioactive Polyphenols: a randomized trial in Nicaraguan children}

Arthur Dover, MD, DTMH ${ }^{1}$; Neema Patel, BS²; KT Park, MD, MS ${ }^{3-4}$

Affiliations: ${ }^{1}$ Aptos Travel Clinic, Aptos, CA; ${ }^{2}$ LiveLeaf, Inc., San Carlos, CA; ${ }^{3}$ Division of Gastroenterology, Hepatology, and Nutrition, Department of Pediatrics, Stanford University School of Medicine; ${ }^{4}$ Center for Health Policy / Primary Care Outcomes Research, Stanford University

Address Correspondence to: Arthur Dover, 9099 Soquel Drive \#6, Aptos, CA, 95003, arthur.dover@gmail.com, Telephone: (831) 818-5632, Fax: (408) 599-3179

Conflicts of Interest and Source of Funding: Dr. Arthur Dover, Dr. KT Park, and Neema Patel consult for LiveLeaf, Inc.

\section{INTRODUCTION}

Diarrhea is the second leading cause of death in children under the ages of 5 years in developing countries (Johansson et al., 2009), a most concerning statistic as diarrhea may be prevented and treated. Acute diarrhea can lead to severe dehydration and electrolyte imbalance by loss of fluids, electrolytes, and nutrients (Munos et al., 2010). Oral rehydration therapy was initially developed to replace cholerainduced fluid loss (Pierce et al., 1969; Sentongo 2004), but has expanded to include diarrhea incited by other pathogens (Hirschhorn 1980; Nalin et al., 1979; Pizarro et al., 1983). The World Health Organization (WHO) standardized an oral rehydration solution (ORS) containing sodium, potassium, chloride, citrate, and glucose (Atia \& Buchman 2009). Although ORS assists in diarrheal management, it does not reduce the duration of diarrhea or fecal volume (Canai et al. 2007). Instead, implementing ORS can increase stool volume in children during acute episodes (Sarker et al., 2001; El-Mougi et al., 1994). In order to optimize efficacy, the WHO recommended a modified ORS with reduced osmolarity, administration of zinc gluconate, non-digestible carbohydrates, rice powder, and probiotic bacteria - all with mixed results (Gregogio et al., 2007; Basu et al., 2007; Narayanappa 2008; Hoekstra et al., 2004; Passariello et al., 2011). 
34 In developing countries, attempts for rehydration using readily available household beverages often

.

exacerbate intestinal fluid loss by elevating osmotic load and disrupting water and electrolyte absorption (Munos et al., 2010; Sentongo 2004). However, the proper use of ORS and public health measures in Nicaragua including widespread rotavirus vaccinations in infants has been associated with a $35 \%$ reduction in childhood mortality over 5 years in the early 1980 s. This rate has since remained relatively constant (Gibbons, Dobie \& Krieger 1994). Currently, antibiotics serve a very limited role in treating diarrhea in children and the utility of anti-motility agents is either contra-indicated or controversial due to heightened infection risks and adverse effects.

The use of naturopathic medicines in rural or developing populations is often attributed to the inaccessibility of western medicines for common infectious illnesses and a traditional belief in the natural, beneficial properties of plant and plant-derived products. Recent investigations into the efficacy of various plants have identified that their phytochemicals can affect intestinal function and motility (Njume \& Goduka 2012; Bukhari et al., 2013; Velazquez et al., 2012; Rajan et al., 2012; Patil, et al., 2012; Ezeja et al., 2012) and provide antibacterial activity (ABbassi \& Hani 2012; Knipping, Garssen \& van’t Land 2012; Ismail, Sestili \& Akhtar 2012; Mariita et al., 2011; Assam et al., 2010). While commercial extraction and processing of these compounds can reduce their viability, a novel processed plant extract composition, LifeDrops (LiveLeaf Inc., San Carlos, CA), captures the bioactive potential of live plant cells. The LifeDrops solution contains a complete complex of green tea (Camellia sinensis) and pomegranate (Punica granatum) incorporating biologic co-factors key to delivering the full capability of the plants' immune response, termed LiveXtract solution (LX). The mechanism behind LiveXtract solutions is based upon a transient polyphenol reaction common to nearly all higher plants. The site activation of this reaction by the body's enzymes delivers a powerful 
synergy of localized injury protection, toxin neutralization, and attenuation of inflammation that cannot

58 be produced by conventional. polyphenol extracts (Romier et al., 2009; Vauzour, et al., 2010; Taylor,

59 Hamilton-Miller \& Stapleton 2005; Biasi et al., 2011; Romier-Crouzet et al., 2009; Kim, Rajalah \&

$60 \mathrm{Wu} 2008)$.

61

62 The objective of this study is to compare the efficacy of ORS+LX (LifeDrops) versus ORS+water

63 (placebo) in reducing the incidence and frequency of loose stools and associated gastrointestinal

64 symptoms of pediatric patients with acute diarrhea in Nicaragua. We hypothesized that the addition of

65 LifeDrops to standard ORS, compared to ORS alone, would reduce the time to normalization of stools 66 and digestive function.

68 MATERIALS \& METHODS

69 Study Design

71 This randomized, double-blinded, placebo-controlled study was conducted at a government-funded

72 community health clinic in Managua, Nicaragua, between August and December 2010. Following

73 torrential rains and flooding in the region from tropical storms Agatha and Matthew, there was a

74 substantial increase in the incidence of consultations for acute diarrhea. With approval of the

75 institutional review board of the Universidad Centroamericana de Ciencias Empresariales (IRB

76 2010013, registered ISRCTN57765025)), treatment-naïve, previously healthy pediatric patients

77 between 2 and 17 years of age who arrived at the clinic with uncontrolled acute diarrhea within 48

78 hours prior to presentation were enrolled in the study. Written informed consent was obtained from the

79 parents or legal guardians of patients who met the inclusion criteria. 


\section{Statistical Analysis}

82 Sample size calculations were based on studies in acute diarrhea using standard ORS treatment in non-

83 cholera pediatric patients. A sample size of $\geq 30$ patients per arm was based upon detecting at least a

$8415 \%$ difference in the duration of diarrhea at the $5 \%$ significance level with $80 \%$ power. Differences

85 between means of parametric data were analyzed with the Student's $t$-Test, with significance set at 0.05

86 level. Nonparametric data were analyzed with Chi-squared and Wilcoxon rank-sum tests.

88 Study Inclusion

89 All patients who presented to the clinic were assessed and included if they had acute gastroenteritis,

90 including diarrhea, for 48 hours or less. Diarrhea was defined as three or more loose or liquid stool per

91 day. Patients were excluded from the study if they had a history of uncontrolled emesis, grossly

92 bloody stool, fever, clinical signs of a coexisting acute systemic illness (e.g., meningitis, sepsis,

93 pneumonia), underlying chronic disease (e.g., heart disease, cystic fibrosis, diabetes), food allergies or

94 other chronic gastrointestinal diseases, admitted use of probiotic agents in the previous 3 weeks or

95 antibiotics or anti-diarrheal medication including over-the-counter and herbal substances in the

96 previous 2 weeks, generalized cachexia, any signs of internal bleeding or drug abuse, or any condition

97 assessed by standard of care to place unnecessary risk if placed on ORS alone. Every patient had a

98 microscopic stool evaluation at the time of enrollment, and those positive for an intestinal protozoan

99 infection were excluded from the study.

\section{ORS + LX vs. ORS+water}

102 After study eligibility was determined and consent was obtained, patients were randomized to one of

103 two arms based upon a computer-generated random number listing. The study arm consisted of

104 ORS+LX (LifeDrops) on day 1, then ORS+water on day 2. The control arm consisted of ORS+water 
105 on day 1, then ORS+LX on day 2. Patients in both arms were given one of the blinded solutions on the

106 first day of clinical evaluation and subsequently monitored by clinic staff for two hours (Figure 1).

107 While not a true cross-over study design, patients were given the solution on day 2 that was opposite of

108 what was provided on day 1 in order to assess if there were any differences in symptom resolution. A

109 graduated dosing scale, based on patients' weight, determined the volume of LiveXtract solution

110 administered (Table 1). In the control arm, the same volume of water was added to the ORS in order to

111 equal the $25 \mathrm{~mL}$ total fluid volume given to patients in the study arm. To enhance the uptake of the test

112 solutions, the ORS contained an added commercial artificial flavor and coloring produced by the

113 Acama company in Central America. Zinc gluconate was not administered during the study period.

115 Two hours after administration of either solution on day 1, the patients were released from the clinic

116 with a maintenance amount of ORS for the next 24 hours. All patients were asked to return within 24

117 hours on day 2 for administration of the alternate solution.

119 Outcome Measures

120 The primary outcome measure was the time elapsed from the initial ingestion of ORS+LX or

121 ORS+water to any subsequent "unformed" stool, based on the Bristol Stool Scale (BSS), a validated

122 method of visually categorizing stool in 7 appearances based on stool shape and consistency. It has

123 been shown to have reproducibility in pediatric cohorts (Lane et al., 2011; Lewis \& Heaton 1997). We

124 considered any BSS $>4$ to be "unformed" and $\leq 4$ to be "formed." The clinical staff ranked the stool

125 during the first 2 hours after solution ingestion and parents were trained to score and report the ranking 126 of each bowel movement while away from clinic. 
128 The secondary outcome measures were defecation urgency and bloating/gas following fluid

129 consumption, and a qualitative rating of abdominal pain (for patients able to comprehend and follow

130 directions) on a numeric scale of 0 (none) to 10 (worst imaginable/continual) at 30, 60, 90, and 120

131 minutes after consumption of either solution on both day 1 and day 2 .

\section{RESULTS}

\section{Patient Demographics}

135 A total of 61 patients were enrolled in this study with 30 patients randomized to the study arm 136 (ORS+LX) and 31 patients to the control arm (ORS+water) on day 1. All subjects were found to be

137 free of protozoan infection by microscopic stool examination, but the specific etiologies of their

138 diarrhea were not definitely known, as per standard of care in this clinical care setting. The patients in

139 each arm were comparable in age (mean age of 8 vs. 7 years, $p=0.51$ ) and weight (mean weight of 27

140 vs. $32 \mathrm{~kg}, \mathrm{p}=0.31$ ), but with more females present in the study arm and more males in the control arm

141 (Table 2).

\section{Response to Solutions Consumed on Day 1}

144 The summary of results shown in Figure 2 demonstrates that patients in the study arm achieved a time-

145 to-last unformed stool (a BSS ranking of 4 or less) in a mean elapsed time of 3.1 hours versus 9.3 hours

146 among patients in the control arm ( $\mathrm{p}=0.002)$ on day 1 of the study. In the study arm, $60 \%$ of the

147 patients had their first bowel movement with a BSS of 4 or less after consuming the ORS+LX. In the

148 control arm, only $29 \%$ of the patients had their first bowel movement with a BSS of 4 or less after

149 ORS+water consumption. At the second movement on day $1,82 \%$ of patients in the study arm versus

$15035 \%$ of patients in the control arm reported stools with a BSS rating of 4 or less. $(\mathrm{p}<0.001)$ 
152 Patients in the study arm also experienced a longer mean time between bowel movements after solution

153 consumption: 3.7 hours in the study arm and 2.8 hours in the control arm, which did not achieve

154 statistical significance. The mean time between the first and second bowel movements after

155 consumption was 7 hours in the study arm versus 4.4 hours in the control group $(\mathrm{p}=0.02)$.

157 Response to Solutions Consumed on Day 2

158 When patients returned on day 2 of the study, those in the study arm received ORS+water while those

159 in the control arm received ORS+LX. After 2 hours, all patients in the study arm reported stool with a

160 BSS rating of 4 or lower. Patients in the control arm subsequently reported resolution of their diarrhea

161 at a rate comparable to that noted on day 1 for patients in the study arm (Figure 2). On day 2, patients

162 in control arm had a mean ranking of stool of 4.5 prior to consuming the ORS $+\mathrm{LX}$, which decreased to

1633.2 by the first bowel movement after consumption and further decreased 2.2 by the end of day 2

$164(\mathrm{p}<0.01)$. Patients given ORS+water on day 1 had a mean number of 4 bowel movements that declined

165 to a mean of 2 after receiving ORS + LX on day $2(\mathrm{p}<0.01)$.

166

167 Secondary Outcome Measures

168 Patient-reported responses (e.g., abdominal pain) were incompletely collected during November and

169 December of 2010, resulting in responses from only 10 study arm patients and 7 control arm patients,

170 sample sizes too small for meaningful analyses. The rating of gas and bloating was comparable

171 between the two arms over the two days, but patients in the control arm did report improvement in their

172 levels of abdominal pain and urgency of defecation soon after consumption of the ORS+LX on day 2

173 (Figures 3 and 4). The rating of abdominal pain in patients in the control arm decreased to levels

174 comparable to that reported by patients in the study arm within 2 hours after consumption of ORS $+\mathrm{LX}$

175 and was essentially identical to patients in the study arm at the end of the study period (Figure 3). The 
176 rating of defecation urgency, despite remaining unchanged for 24 hours after consumption of

177 ORS+water, declined substantially within 60 minutes post-ORS+LX consumption and continued to

178 decline during the study period (Figure 4). No adverse events were reported or observed during the

179 study due to ingestion of either of the solutions, and none were reported to the clinic staff after the

180 conclusion of the study period. Additionally, relapse of symptoms was not subsequently reported to the

181 clinic staff.

182

\section{DISCUSSION}

184 In this randomized controlled trial, we demonstrate that compared to ORS alone, supplementation of a 185 novel LiveXtract solution (LifeDrops) significantly decreased resolution time of acute diarrhea and 186 accelerated normalization of stool consistency. All patients in the study experienced faster resolution of 187 their diarrhea after receiving ORS+LX, and all soon achieved normalization of stool consistency. The 188 intervention cohort receiving ORS $+\mathrm{LX}$ had normalization to $\mathrm{BSS} \leq 4$ stool consistency and frequency

189 by the end of day 1 . Similarly, control patients who received ORS+LX on day 2 (after receiving

190 ORS+water on day 1) reported comparable efficacy by the end of day 2.

192 Secondary outcome measures of abdominal pain and defecation urgency also improved for both 193 cohorts upon initiation of ORS+LX by the end of the same day. By the end of the monitoring period on 194 day 2, patients in the control cohort noted a reduction in both adverse symptoms similar to patients in 195 the intervention cohort reported by the end of monitoring on day 1. No adverse events were reported or 196 observed in any patient receiving ORS + LX.

198 One limitation of our study is the lack of infectious pathogen identification in subjects' acute diarrheal

199 illness. This study was conducted at a government-funded community health clinic in Managua, 
200 Nicaragua following torrential rains and flooding in this region in late 2010. Resource limitations and

201 prioritization of streamlined humanitarian efforts made pathogen identification difficult in the context

202 of a clinical trial, although subjects with evidence of any protozoa by light microscopy were excluded

203 and referred for treatment. Given our hypothesis that the LiveXtract solution maintained the natural

204 antibacterial properties of Camellia sinensis and Punica granatum within the enteric tract after

205 consumption, we theorize that plant extracts rich in polyphenols have the potential to stimulate innate

206 host immune processes by action of phyto chemicals from natural plant immunity and to antagonize

207 common enteric pathogens responsible for acute bacterial and viral gastroenteritis. Previous literature

208 has identified waterborne enteric pathogens as likely gram negative bacterial species, such

209 enterotoxigenic Aeromonas, Campylobacter, Salmonella, Shigella, and enterotoxigenic Escherichia

210 coli, which all thrive in warm freshwater environments (Burke et al., 1983; Ashbolt 2004), reproduced

211 in the natural elements present in our study.

212

213 Another limitation is that our data represent a snapshot of a narrow study timeframe and one specific

214 geographical location. While acknowledging the weaknesses of our study, we also recognize the

215 strength of our study's randomized study design. For a prospective pilot study, we surpassed adequate

216 enrollment numbers to show a clear statistical difference between ORS+LX vs. ORS+water. Among

217 the individual subjects, we showed distinct reproducibility of the treatment effect upon introduction of

218 ORS+LX between individual patients.

220 The limited number of pediatric patients who provided data for secondary outcome measures did not

221 permit statistical analyses of the changes in these patients' quality of life. However, the data do show a

222 trend of reducing abdominal pain and defecation urgency with consumption of the polyphenol

223 supplement, which needs to be verified in future clinical outcome studies. 
225 Preventing and reducing morbidity and mortality from acute diarrheal illnesses causing dehydration is 226 a significant public health concern, and remains an on-going global health initiative. Although the use 227 of ORS to restore intravascular fluid losses remains the standard of care in most clinical scenarios, 228 there are limited clinical alternatives aimed to actively shorten the time of acute diarrheal fluid and 229 electrolyte losses. LiveLeaf LifeDrops solution potentially represents a novel approach to effectively 230 reduce morbidity and mortality from acute diarrhea illnesses in certain situations. In this preliminary 231 study, we report the results of the first prospective clinical trial using this unique supplement to ORS.

232 Published literature in this area includes several negative studies of the addition of rice or non-

233 digestible carbohydrates to ORS (Sarker et al., 2001; El-Mougi et al., 1994; Hoekstra et al., 2004;

234 Faruque et al., 1997; Khan et al., 2005). Further literature review of the efficacy of trace elements such 235 as zinc (Gregorio et al., 2007; CHOICE Study Group 2001) and probiotics (Basu et al., 2007; Wadhwa 236 et al., 2011) to reduce acute diarrheal disease burden are mixed. The current recommendation of the

237 World Health Organization (WHO) is to provide low osmolarity ORS and zinc supplementation for 10 238 to 14 days (Burke et al., 1983), which is associated with reduced time to resolution of diarrhea in 239 several clinical studies (El-Mougi et al., 1994; Gregorio et al., 2007; Boran et al., 2006; Dutta et al., 240 2000; Patel, Badhoniya \& Dibley 2013), but with times substantially longer than the 3 hours noted in 241 patients given the LiveXtract solution.

243 Future directions should be aimed at understanding the mechanisms of phytochemicals as potential 244 consumable agents effective in acute infectious gastroenteritis. Elucidation of the molecular basis of 245 the phytochemicals' action on enteric pathogens - through a detailed biochemical pathway - should be 246 pursued, as well as their possible interaction with innate host intestinal immune systems, supported by 
microbiota analysis. Clinical research efforts should also be directed to test the robustness of our initial

248 efficacy data through reproducibility while subject to contextual study variability.

CONCLUSION

251 In this randomized clinical study, pediatric patients with acute diarrhea experienced rapid improvement 252 of stool consistency following ingestion of the LiveXtract solution. Further clinical data are necessary

253 in order to corroborate these results, but the rapid resolution in pediatric patients in this study suggests

254 a well-tolerated, safe, and effective option for the resolution of acute diarrhea syndrome.

\section{ACKNOWLEDGEMENTS}

257 We thank Dr. Telma Noguera (Instituto Centroamericano de Investigación Clínica, Managua, 258 Nicaragua) and Mr. Rob Wotring (LiveLeaf Inc.) for their contribution in coordination of data 259 collection.

\section{REFERENCES}

262

263

264
Abbassi F, Hani K. 2012. In vitro antibacterial and antifungal activities of Rhus tripartitum used as antidiarrheal in Tunisian folk medicine. Natural Products Research 26(23): 2215-2218.

Ashbolt NJ. 2004. Microbial contamination of drinking water and disease outcomes in developing regions. Toxicology 198(1-3): 229-238.

Assam AJ, Dzoyem JP, Pieme CA, Penlap VB. 2010. In vitro antibacterial activity and acute toxicity studies of aqueous-methanol extract of Sida rhombifolia Linn. (Malvaceae). BMC Complementary and Alternative Medicine 10: 40-46.

Atia AN, Buchman AL. 2009. Oral rehydration solutions in non-cholera diarrhea: a review. American Journal of Gastroenterology 104(10): 2596-2604.

Basu S, Chatterjee M, Ganguly S, Chandra PK. 2007. Effect of Lactobacillus rhamnosus GG in persistent diarrhea in Indian children: a randomized controlled trial. Journal of Clinical Gastroenteroogy 41(8): 756-760. 
Biasi F, Astegiano M, Maina M, Leonarduzzi G, Poli G. 2011. Polyphenol supplementation as a complementary medicinal approach to treating inflammatory bowel disease. Current Medicinal Chemistry 18(31): 4851-4865.

Boran P, Tokuc G, Vagas E, Oktem S, Gokduman MK. 2006. Impact of zinc supplementation in children with acute diarrhoea in Turkey. Archives of Diseases in Childhood 91: 296-299.

Bukhari IA, Shah AJ, Khan RA, Gilani AH. 2013. Gut modulator effects of Conya bonariensis explain its traditional use in constipation and diarrhea. European Review for Medical and Pharmacological Sciences 17(4): 552-528.

Burke V, Gracey, M, Robinson J, Peck D, Beaman J, Bundell C. 1983. The microbiology of childhood gastroenteritis: Aeromonas species and other infective agents. Journal of Infectious Diseases 148(1): 68-74.

Canai RB, Cirillo P, Terrin G, Cesarano L, Spagnuolo MI, DeVincenzo A, Albano F, Passariello A, DeMarco G, Manquso F, Guarino A. 2007. Probiotics for treatment of acute diarrhoea in children: randomised clinical trial of five different preparations. British Medical Journal. 335(7615): 340.

CHOICE Study Group. 2001. Multicenter, randomized, double-blind clinical trial to evaluate the efficacy and safety of a reduced osmolarity oral rehydration salts solution in children with acute watery diarrhea. Pediatrics 107(4): 613-618.

Dutta P, Mitra U, Dutta A, Niyogi SK, Dutta S, Manna B, Basak M, Mahapatra TS, Bhattacharya SK. 2000. Impact of zinc supplementation in malnourished children with acute watery diarrhoea. Journal of Tropical Pediatrics 46(5): 259-263.

El-Mougi M, el-Akkad N, Hendawi A, Hassan M, Amer A, Fontaine O, Pierce NF. 1994. Is a lowosmolarity ORS solution more efficacious than standard WHO ORS solution? Journal of Pediatric Gastroenterology and Nutrition 19(1): 83-86.

Ezeja IM, Ezeigbo II, Madubuike KG, Udeh NE, Ukweni IA, Akomas SC, Ifenkwe DC. 2012. Antidiarrheal activity of Pterocarpus erinaceus methanol leaf extract in experimentally-induced diarrhea. Asian Pacific Journal of Tropical Medicine 5(2): 147-150.

Faruque AS, Hoque SS, Fuchs GJ, Mahalanabis D. 1997. Randomized, controlled, clinical trial of rice versus glucose oral rehydration solutions in infants and young children with acute watery diarrhoea. Acta Paediatrica 86(12): 1308-1311.

Gibbons E, Dobie SA, Krieger J. 1994. Evaluation of oral rehydration therapy in Matiguas, Nicaragua. Public Health Report 109(3): 428-433.

Gregorio GV, Dans LF, Cordero CP, Panelo CA. 2007. Zinc supplementation reduced cost and duration of acute diarrhea in children. Journal of Clinical Epidemiology 60(6): 560-566.

Hirschhorn N. 1980. The treatment of acute diarrhea in children: an historical and physiological perspective. American Journal of Clinical Nutrition 33(3): 637-663. 
Hoekstra JH, Szajewska H, Zikri MA, Micetic-Turk D, Weizman Z, Papadopoulou A, Guarino A, Dias JA, Oostvogels B. 2004. Oral rehydration solution containing a mixture of non-digestible carbohydrates in the treatment of acute diarrhea: a multicenter randomized placebo controlled study on behalf of the ESPGHAN Working Group on Intestinal Infections. Journal of Pediatric Gastroenterology and Nutrition 39(3): 239-245.

Ismail T, Sestili P, Akhtar S. 2012. Pomegranate peel and fruit extracts: a review of potential antiinflammatory and anti-infective effects. Journal of Ethnopharmacology 143(2): 397-405.

Johansson EW, Wardlaw T, Binkin N. 2009. Diarrhoea: Why Children Are Still Dying and What Can Be Done. New York, New York: UNICEF and Geneva, Switzerland: World Health Organization.

Khan AM, Sarker SA, Alam NH, Hossain MS, Fuchs GJ, Salam MA. 2005. Low osmolar oral rehydration salts solution in the treatment of acute watery diarrhoea in neonates and young infants: a randomized, controlled clinical trial. Journal of Health, Population and Nutrition 23(1): 52-57.

Kim HR, Rajalah R, Wu QL. 2008. Green tea protects rats against autoimmune arthritis by modulating disease-related immune events. Journal of Nutrition 138(11): 2111-2116.

Knipping K, Garssen J, van't Land B. 2012. An evaluation of the inhibitory effects against rotavirus infection of edible plant extracts. Virology Journal 26; 9: 137-144.

Lane MM, Czyzewski DI, Chumpitazi BP, Shulman RJ. 2011. Reliability and validity of a modified Bristol Stool Form Scale for children. The Journal of Pediatrics 159(3): 437-441.

Lewis SJ, Heaton KW. 1997. Stool form scale as a useful guide to intestinal transit time. Scandinavian Journal of Gastroenterology 32(9): 920-924.

Mariita RM, Orodho JA, Okemo PO, Kirimuhuzya C, Otieno JN, Magadula JJ. 2011. Methanolic extracts of Aloe secundiflora Engl. inhibits in vitro growth of tuberculous and diarrhea-causing bacteria. Pharmacognosy Research 3(2): 95-99.

Munos MK, Fischer Walker CL, Black RE. 2010. The effect of oral rehydration solution and recommended home fluids on diarrhoea mortality. International Journal of Epidemiology 39(suppl 1): i75-i87.

Nalin DR, Levine MM, Mata L, de Cespedes C, Vargas W, Lizano C, Loria AR, Simhon A, Mohs E. 1979. Oral rehydration and maintenance of children with rotavirus and bacterial diarrheas. Bulletin of the World Health Organization 57(3): 453-459.

Narayaappa D. 2008. Randomized double blinded controlled trial to evaluate the efficacy and safety of Bifilac in patients with acute viral diarrhea. Indian Journal of Pediatrics 75(7): 709-713.

Njume C, Goduka NI. 2012. Treatment of diarrhoea in rural African communities: an overview of measures to maximize the medicinal potentials of indigenous plants. International Journal of Environmental Research and Public Health 9(11): 3911-3933. 
Passariello A, Terrin G, De Marco G, Cecere G, Ruotolo S, Marino A, Cosenza L, Tardi M, Nocerino R, Berni Canani R. 2011. Efficacy of a new hypotonic oral rehydration solution containing zinc and prebiotics in the treatment of childhood acute diarrhea: a randomized controlled study. Journal of Pediatrics 158(2): 288-289.

Patil VV, Bhangale SC, Chaudhari KP, Kakade RT, Thakare VM, Bonde CG, Patil VR. 2012. Evaluation of the antidiarrheal activity of the plant extracts of Ficus species. Journal of Chinese Integrative Medicine 10(3): 347-352.

Patel AB, Badhoniya N, Dibley MJ. 2013. Zinc and copper supplementation are not cost-effective interventions in the treatment of acute diarrhea. Journal of Clinical Epidemiology 66(2013): 52-61.

Pierce NF, Sack RB, Mitra RC, Banwell JG, Brigham KL, Fedson DS, Mondal A. 1969. Replacement of water and electrolyte losses in cholera by an oral glucose-electrolyte solution. Annals of Internalpi Medicine 70(6): 1173-1176.

Pizarro D, Posada G, Villavicencio N, Mohs E, Levine MM. 1983. Oral rehydration in hypernatremic and hyponatremic diarrheal dehydration. American Journal of Diseases of Children 137(8): 730-734.

Rajan S, Suganya H, Thirunalasundari T, Jeeva S. 2012. Antidiarrheal efficacy of Mangifera indica seed kernel on Swiss albino mice. Asian Pacific Journal of Tropical Medicine 5(8): 630-633.

Romier B, Schneider YJ, Larondelle Y, During A. 2009. Dietary polyphenols can modulate the intestinal inflammatory response. Nutrition Reviews 67(7): 363-378.

Romier-Crouzet B, Van De Walle J, During A, Joly A, Rousseau C, Henry O, Larondelle Y, Schneider YJ. 2009. Inhibition of inflammatory mediators by polyphenolic plant extracts in human intestinal Caco-2 cells. Food and Chemical Toxicology 47(6): 1221-1230.

Sarker SA, Mahalanabis D, Alam NH, Sharmin S, Khan AM, Fuchs GJ. 2001. Reduced osmolarity oral rehydration solution for persistent diarrhea in infants: a randomized controlled clinical trial. Journal of Pediatrics 138(4): 532-538.

Sentongo TA. 2004. The use of oral rehydration solutions in children and adults. Current Gastroenterology Reports 6(4): 307-313.

Taylor PW, Hamilton-Miller JM, Stapleton PD. 2005. Antimicrobial properties of green tea catechins. Food Science and Technology Bulletin 2: 71-81.

Vauzour D, Rodriguez-Mateos A, Corona G, Oruna-Concha MJ, Spencer JP. 2010. Polyphenols and human health: prevention of disease and mechanisms of action. Nutrients 2(11): 1106-1131.

Velazquez C, Correa-Basurto J, Garcia-Hernandez, Barbosa E, Tesorno-Cruz E, Calzada S, Calzada F. 2012. Anti-diarrheal activity of (-)-epicatechin from Chiranthodendron pentadactylon Larreat: experimental and computational studies. Journal of Ethnopharmacology 143(2); 716-719. 
420 Wadhwa N, Natchu UC, Sommerfelt H, Strand TA, Kapoor V, Saini S, Kainth US, Bhatnagar S. 2011.

421 ORS containing zinc does not reduce duration or stool volume of acute diarrhea in hospitalized

422 children. Journal of Pediatric Gastroenterology and Nutrition 53(2): 161-167.

Figure 1. Study design and patient disposition. Patients randomized to the Study Arm were given a mixture of oral rehydration salts (ORS) and LiveXtract (LX) solution (test solution) on day 1 and then a mixture of ORS and water (placebo) on day 2. Patients randomized to the Control Arm were given a mixture of ORS and water on day 1 and then a mixture of ORS and LiveXtract solution on day 2.

Figure 2. Mean time (hours) to resolution of acute diarrhea following consumption of either a mixture of oral rehydration salts (ORS) and LiveXtract (LX) solution (test solution) or a mixture of ORS and water (placebo) on day 1 and day 2 of the study. On day 1 , the mean times to resolution were significantly different ( $\mathrm{p}=0.002)$.

Figure 3. Mean ranking of abdominal pain over two days at 30 minute intervals, after consuming either a mixture of oral rehydration salt (ORS) and LiveXtract (LX) solution (study arm) or ORS mixed with water (control arm). Pain was ranked between 0 (no pain) and 10 (worst pain imaginable).

Figure 4. Mean ranking of urgency to defecate over two days at 30 minute intervals, after consuming either a mixture of oral rehydration salt (ORS) and LiveXtract (LX) solution (study arm) or ORS mixed with water (control arm). Urgency was ranked between 0 (none) and 10 (unable to control). 


\section{Table $\mathbf{1}$ (on next page)}

Serving size of LiveXtract solution administered based upon the weight of the patient. 
4 Table 1. Serving Size of LiveXtract Solution Administered based upon the weight of the patient.

5

\begin{tabular}{cc}
\hline Weight of Patient, $\mathrm{kg}$ & Serving Size, $\mathrm{mL}$ \\
\hline 10 to 19 & 3.5 \\
20 to 29 & 7.0 \\
30 to 39 & 10.5 \\
40 to 49 & 14.0 \\
50 to 59 & 17.5 \\
\hline
\end{tabular}

6

7 
Table 2 (on next page)

Demographics of study population given oral rehydration solution and water (ORS+water) and oral rehydration solution and LiveXtract solution (ORS+LX). 
5 Table 2. Demographics of study population given oral rehydration solution and water (ORS+water) and oral 6 rehydration solution and LiveXtract solution (ORS+LX).

7

\begin{tabular}{cccc}
\hline Demographics & $\begin{array}{c}\text { Study Arm (n=30) } \\
\text { (ORS + LX) }\end{array}$ & $\begin{array}{c}\text { Control Arm (n=31) } \\
(\text { ORS + Water })\end{array}$ & $P$ \\
\hline Age, mean (SD), years & $8(5.33)$ & $7(5.53)$ & $0.51^{\mathrm{a}}$ \\
Weight, mean (SD), kg & $32(19.89)$ & $27(19.32)$ & $0.31^{\mathrm{a}}$ \\
Sex (Male/Female) & $13 / 17$ & $18 / 13$ & $0.16{\text { (Study arm })^{\mathrm{b}}}^{\mathrm{b}}$ \\
& & & $0.11($ Control arm) \\
\hline
\end{tabular}

8

9 a Student's t-test, significance set at 0.05

10 b Chi-squared test, significance set at 0.05

11

12 


\section{1}

Study design and patient disposition.

Patients randomized to the Study Arm were given a mixture of oral rehydration salts (ORS) and LiveXtract (LX) solution (test solution) on day 1 and then a mixture of ORS and water (placebo) on day 2. Patients randomized to the Control Arm were given a mixture of ORS and water on day 1 and then a mixture of ORS and LiveXtract solution on day 2.

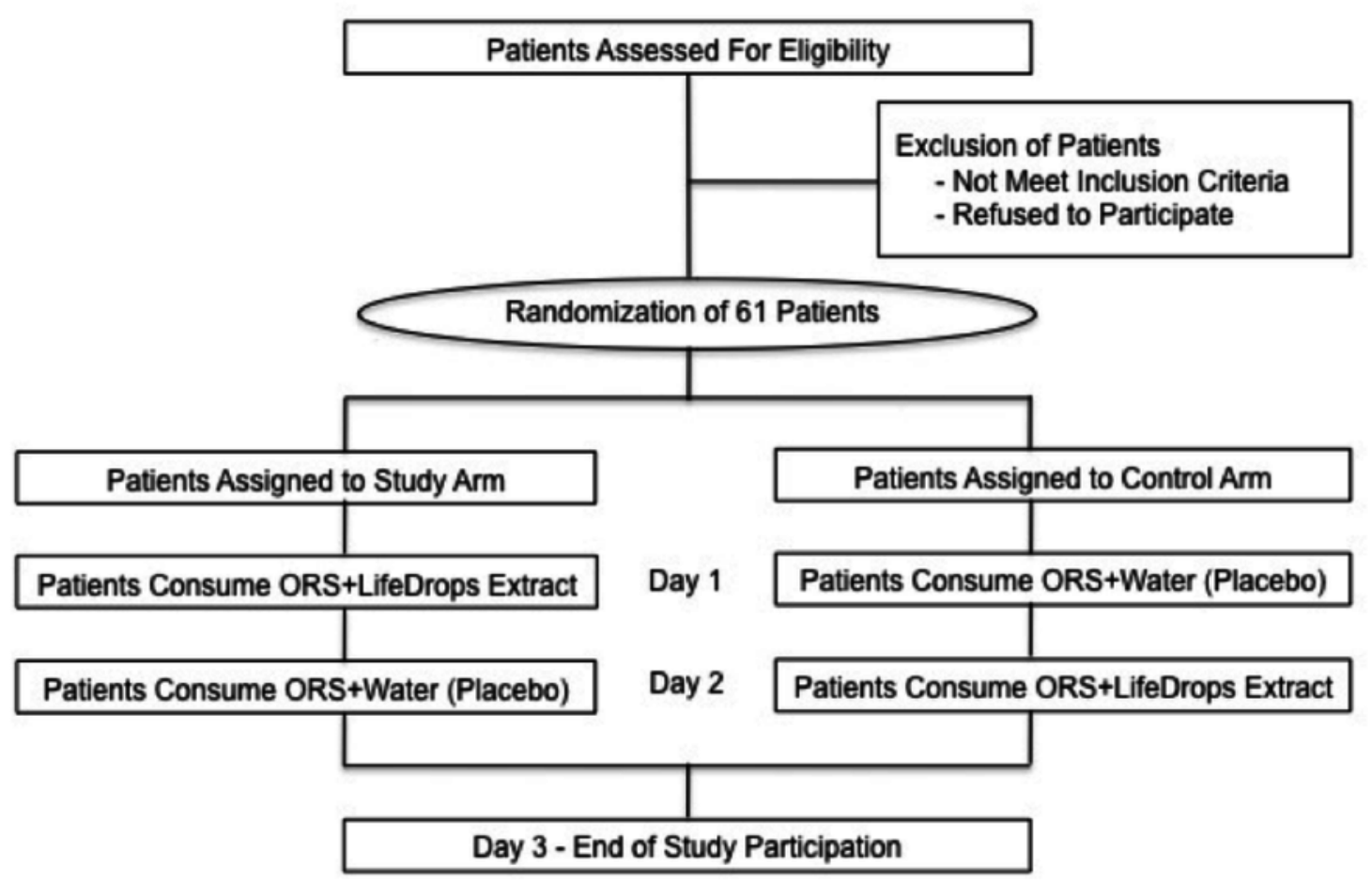


2

Mean time (hours) to resolution of acute diarrhea following consumption of either a mixture of oral rehydration salts (ORS) and LiveXtract (LX) solution (test solution) or a mixture of ORS and water (placebo) on day 1 and day 2 of the study.
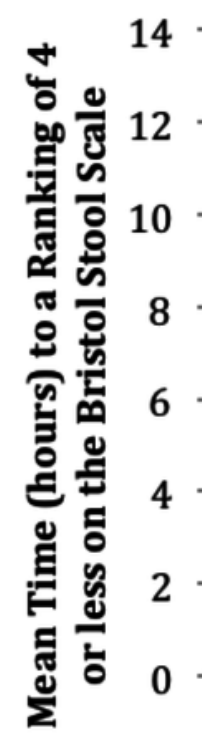

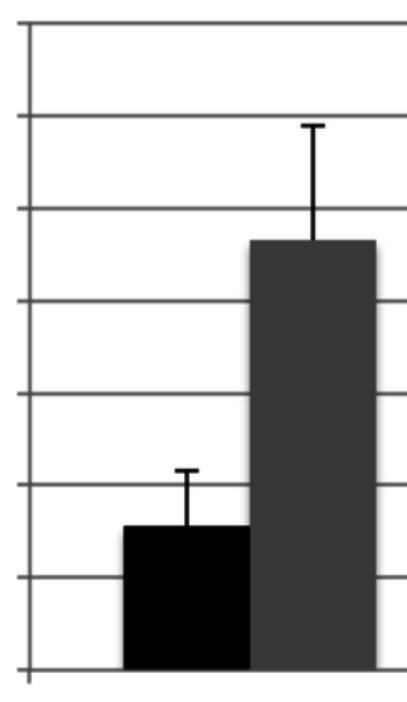

Day 1

\section{Time of monitoring}

- ORS+LX on Day 1; ORS +water on Day 2

ORS+water on Day 1; ORS

+ LX on Day 2 
3

Mean ranking of abdominal pain over two days at 30 minute intervals, after consuming either a mixture of oral rehydration salt (ORS) and LiveXtract (LX) solution (study arm) or ORS mixed with water (control arm).

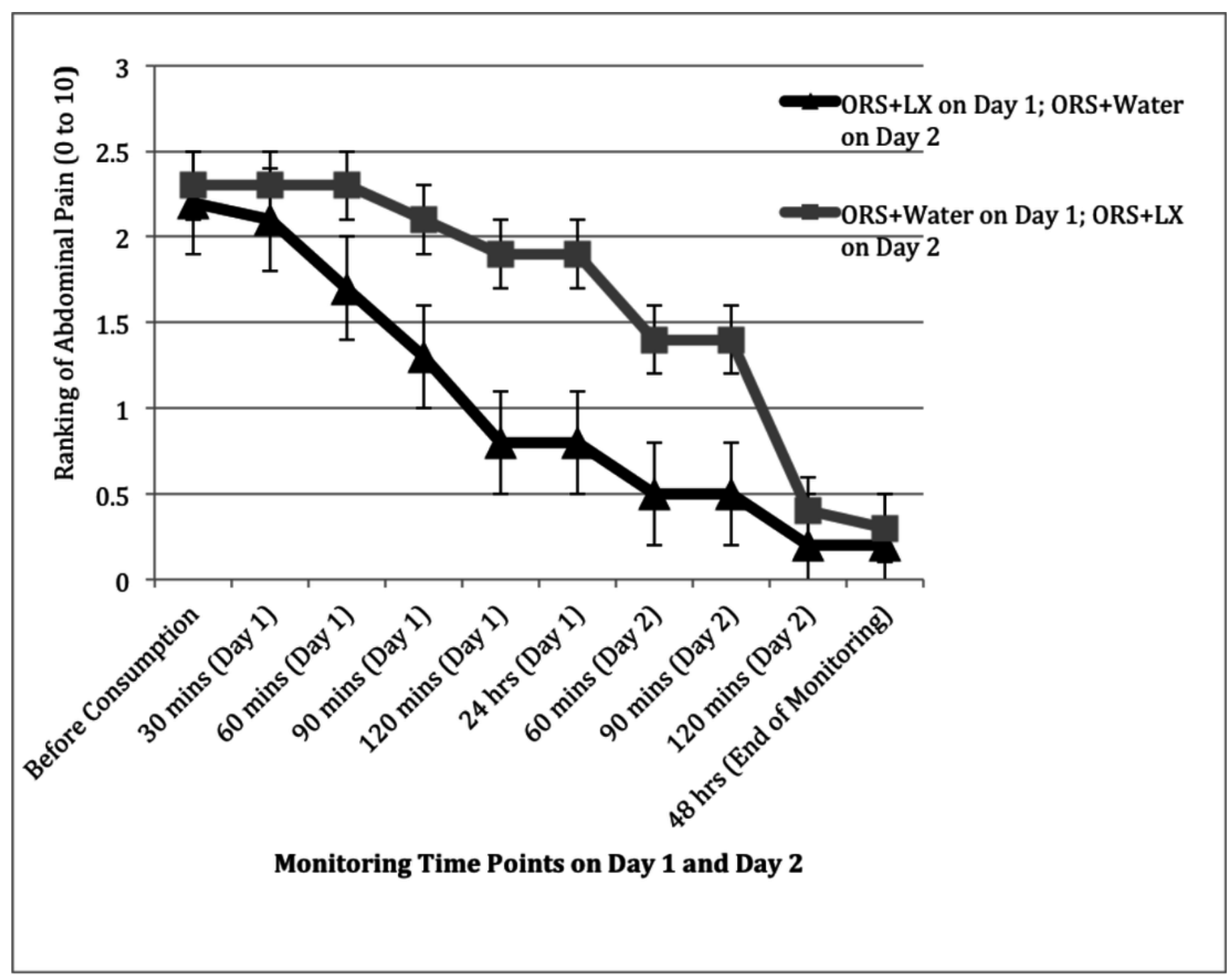


4

Mean ranking of urgency to defecate over two days at 30 minute intervals, after consuming either a mixture of oral rehydration salt (ORS) and LiveXtract (LX) solution (study arm) or ORS mixed with water (control arm).

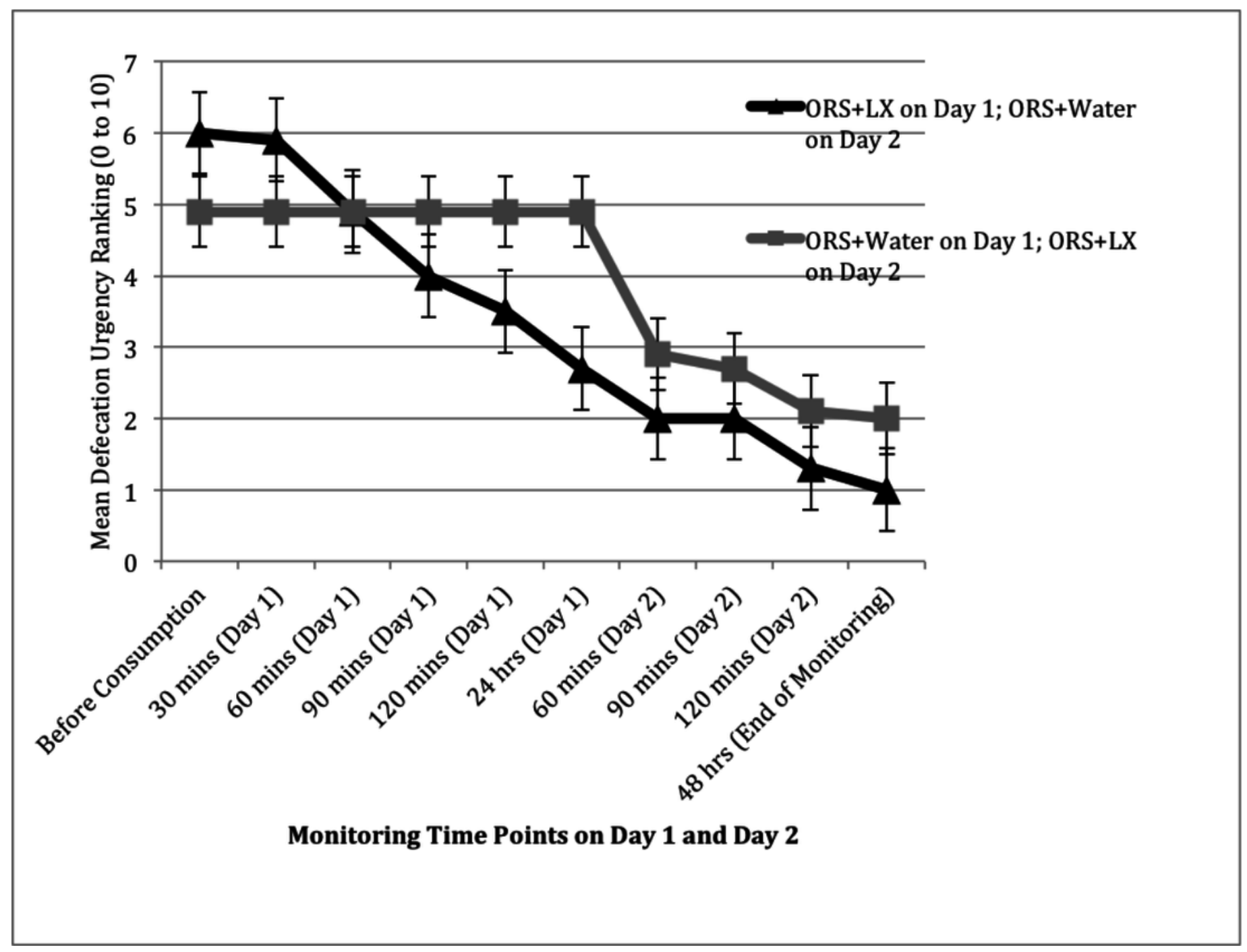

